\title{
Carbon nanotubes in the Coulomb blockade regime
}

\author{
H. Mehrez, ${ }^{1}$ Hong Guo, ${ }^{1}$ Jian Wang, ${ }^{2}$ and Christopher Roland ${ }^{3}$ \\ ${ }^{1}$ Center for the Physics of Materials and Department of Physics, McGill University, Montreal, PQ, Canada H3A 2T8 \\ ${ }^{2}$ Department of Physics, The University of Hong Kong, Pokfulam Road, Hong Kong, China \\ ${ }^{3}$ Department of Physics, The North Carolina State University, Raleigh, North Carolina 27695
}

(Received 29 January 2001; published 4 June 2001)

\begin{abstract}
Quantum transport through finite-length single wall carbon nanotubes was investigated theoretically in the Coulomb blockade regime. The spin-degenerate state of the nanotube is found to play a crucial role, and is responsible for the experimentally observed alternation in the heights of the conductance spectrum as electrons are added to the nanotubes. We also show that the relaxation of the energy eigenstates, which takes place as the electrons tunnel to and from the nanotubes, is responsible for the current saturation as a function of bias voltage polarity.
\end{abstract}

DOI: 10.1103/PhysRevB.63.245410

PACS number(s): 72.80.Rj, 73.23.Ad, 73.61.Wp

\section{INTRODUCTION}

In this paper we report a theoretical analysis of quantum transport through carbon nanotubes (CNT) in the Coulomb blockade (CB) regime. This work is motivated by the impressive experimental data. ${ }^{1-3}$ In particular, recent measurements ${ }^{3}$ on $\mathrm{CNT}$ in the $\mathrm{CB}$ regime showed strong correlation between the zero-bias conductance and the parity of the number of electrons in the tube. The conductance is higher (lower) if the number of electrons in the CNT is even (odd). This low-high peak alternation continues as the gate voltage $\left(V_{g}\right)$ is swept. It was also found that the saturation current is nonsymmetric when the polarity of the bias voltage $\left(V_{b}\right)$ is reversed, indicating nonsymmetric coupling and energy eigenstate rearrangements when an electron enters/ leaves the CNT. Previous theoretical works on transport through CNT were focused on infinite ${ }^{4}$ or finite length ${ }^{5}$ tubes with good contacts. In that case $\mathrm{CB}$ effects are irrelevant. However, no such corresponding analysis exists for CNT in the $\mathrm{CB}$ regime to explain the interesting phenomena reported. ${ }^{3}$

The experimental data ${ }^{3}$ have clearly shown that the oddeven effect is related to the filling of spin states of the CNT which acts as a quantum dot (QD). We therefore believe that this phenomenon should not be special to CNT but has to be more general for the physics of CB. Indeed, experimental data $^{6,7}$ on GaAs QD very weakly coupled to the leads have given indications of peak height alternation ${ }^{8}$ as $V_{g}$ was scanned. But the QD data ${ }^{6,7}$ were less pronounced compared with the CNT data ${ }^{3}$ as far as peak height alternation is concerned. The reason for this discrepancy could be attributed to the randomness of the coupling between the QD eigenstates and the leads as a function of $V_{g}$. Nevertheless, it is an interesting possibility that, for very weakly coupled QD, the odd-even number of electrons in the dot can play an important role in the transport properties of the device in the $\mathrm{CB}$ regime.

The basic theory of transport in CB regime has been well established $^{9}$ under the assumption of independent spin states. ${ }^{10,11}$ When the coupling of a QD to the leads is not extremely weak, fluctuation in the number of electrons in the QD is large enough so that one does not distinguish whether there is an even or odd number of electrons inside the QD. ${ }^{8}$ But to understand the odd-even effect reported in the CNT data, ${ }^{3}$ the independent spin assumption should be extended because it would result in equal peak heights regardless of the number of electrons being odd or even. ${ }^{, 11}$ Changing coupling constants between the leads and QD eigenstates monotonically ${ }^{10}$ can give different peak heights, but this is not correlated to the number of electrons. To investigate this problem, we have extended a recently developed formalism by Xiong and Xiong ${ }^{12}$ where a scattering matrix approach is used to examine the CB problem. We found that the spindegenerate states of the nanotube play a crucial role, and are responsible for the experimentally observed alternation in the heights of the conductance spectrum as electrons are added to the nanotubes. We also found that the relaxation of the energy eigenstates, which takes place as the electrons tunnel to and from the nanotubes, is responsible for the current saturation as a function of bias voltage polarity. Our results are therefore in good qualitative agreement with the experimental observation.

The paper is organized as follows. In the next section we report the theory, the results are contained in the following sections, while the last section is reserved for a short summary.

\section{THEORETICAL FORMALISM}

We have extended the work by Xiong and Xiong, ${ }^{12}$ and our theoretical calculation is based on writing the total manybody wave function as a linear combination of all possible single-particle wave functions and calculating the transmission coefficients. As mentioned above, the results obtained this way are in good qualitative agreement with the experimental data on CNT.

We describe the CNT device using a tight-binding model:

$$
\begin{aligned}
H= & \sum_{m \neq-1,0 ; \sigma} t_{0}\left[a_{m, \sigma}^{\dagger} a_{m+1, \sigma}\right]+\sum_{m \neq 0 ; \sigma}\left(\epsilon_{0}+\eta_{m} e V_{b}\right) a_{m, \sigma}^{\dagger} a_{m, \sigma} \\
& +\sum_{q ; \sigma ; \sigma^{\prime} ; \lambda=L, R}\left[t_{q \sigma \sigma^{\prime}}^{\lambda} a_{-1(1), \sigma^{\prime}}^{\dagger} d_{q, \sigma^{\prime}}+\text { H.c }\right] \\
& +\sum_{q ; \sigma}\left(\xi_{q}+V_{g}\right) d_{q, \sigma}^{\dagger} d_{q, \sigma}+\frac{e^{2}}{2 C}\left(\sum_{q ; \sigma} d_{q, \sigma}^{\dagger} d_{q, \sigma}\right)^{2}
\end{aligned}
$$


where $a_{m, \sigma}^{\dagger}, d_{q, \sigma}^{\dagger}\left(a_{m, \sigma}, d_{q, \sigma}\right)$ correspond to the creation (annihilation) of electrons with spin $\sigma=\uparrow, \downarrow$ of state $m$ in the leads and state $q$ in the CNT, respectively. $t_{0}$ and $t_{q \sigma \sigma^{\prime}}^{\lambda=L(R)}$ denote nearest-neighbor hopping parameters in the leads, and between the left (right) lead with spin $\sigma$ and CNT state with spin $\sigma^{\prime} ; \epsilon_{0}, \xi_{q}$ are the on-site energies in the leads and in CNT; and $\eta_{m}=0,1$ describes the lead to which the bias voltage is applied. We have included the Coulomb charging energy via the $e^{2} / 2 C$ term as described in the constant interaction (CI) picture. From the experimental data ${ }^{3}$ on CNT devices, we infer the charging energy $U=e^{2} / C \simeq 25 \mathrm{meV}$, $\Delta E=\xi_{q+1}-\xi_{q} \simeq 5 \mathrm{meV}$. Similar to the experimental situation we consider CNT very weakly coupled to the leads so that conductance $G \ll G_{0}=e^{2} / h$.

At low temperature $T$ the many-body scattering wave function $|\Psi\rangle$ of the system can be approximated by an expansion on a basis set formed by the lowest single-electron eigenstates. ${ }^{12}$ If there is an even $(=2 M)$ or odd $(2 M+1)$ number of electrons already in the scattering region (the CNT region), we write the even $\left(\left|\Psi^{E}\right\rangle\right) / \operatorname{odd}\left(\left|\Psi^{O}\right\rangle\right)$ wave function separately as

$$
\begin{aligned}
\left|\Psi^{E}\right\rangle= & \sum_{m \neq 0 ; \sigma} p_{m, \sigma} a_{m, \sigma}^{\dagger}\left|\phi_{g}\right\rangle+\sum_{h>M ; \sigma} A_{h, \sigma} d_{h, \sigma}^{\dagger}\left|\phi_{g}\right\rangle, \\
\left|\Psi^{O}\right\rangle= & \sum_{h>M ; \sigma^{\prime}}\left\{\sum_{m \neq 0 ; \sigma} p_{m, \sigma, \sigma^{\prime}} a_{m, \sigma}^{\dagger}+A_{h, \sigma^{\prime}, \bar{\sigma}^{\prime}} d_{h, \bar{\sigma}^{\prime}}^{\dagger}\right. \\
& \left.+\sum_{h^{\prime}>M\left(h^{\prime} \neq h\right) ; \sigma} A_{h, \sigma, \sigma^{\prime}} d_{h^{\prime}, \sigma}^{\dagger}\right\} d_{h, \sigma^{\prime}}^{\dagger}\left|\phi_{g}\right\rangle,
\end{aligned}
$$

where $\left|\phi_{g}\right\rangle \equiv\left\{\Pi_{i=1}^{M} d_{i \uparrow}^{\dagger} d_{i \downarrow}^{\dagger}\right\}|0\rangle$ describes the $2 M$ electrons in the ground state. To obtain coefficients $p$ 's and $A$ 's, we solve $H\left|\Psi^{E(O)}\right\rangle=E\left|\Psi^{E(O)}\right\rangle$. For $m \neq-1,0,1$ we obtain

$\epsilon p_{m \sigma\left(\sigma^{\prime}\right)}=\left(\epsilon_{0}+\eta_{m} V_{b}\right) p_{m \sigma\left(\sigma^{\prime}\right)}+t_{0}\left[p_{m-1 \sigma\left(\sigma^{\prime}\right)}+p_{m+1 \sigma\left(\sigma^{\prime}\right)}\right]$,

where

$$
\epsilon=\left\{\begin{array}{l}
{\left[E-2 \sum_{i=1}^{M} \xi_{i}-2 M V_{g}-(2 M)^{2} \frac{e^{2}}{2 C}\right]} \\
{\left[E-2 \sum_{i=1}^{M} \xi_{i}-\xi_{q}-(2 M+1) V_{g}-(2 M+1)^{2} \frac{e^{2}}{2 C}\right]}
\end{array}\right.
$$

for systems with odd and even number of electrons, respectively. From the above equations we can find the solution for coefficients $p_{m \sigma\left(\sigma^{\prime}\right)}$ :

$$
p_{m \sigma\left(\sigma^{\prime}\right)}=\left\{\begin{array}{ll}
e^{i k^{L}(m+1)}+r_{\sigma\left(\sigma^{\prime}\right)} e^{-i k^{L}(m+1)} & m \leqslant-1 \\
t_{\sigma\left(\sigma^{\prime}\right)} e^{i k^{R}(m-1)} & m \geqslant 1
\end{array},\right.
$$

where $r_{\sigma\left(\sigma^{\prime}\right)}$ and $t_{\sigma\left(\sigma^{\prime}\right)}$ are the reflection and transmission coefficients for the state with wave vectors $k^{L(R)}$ in the left (right) lead. These states satisfy the energy dispersion relation in the leads, $\epsilon-\epsilon_{0}-\eta^{L(R)} V_{b}=2 t_{0} \cos \left(k^{L(R)}\right)$, where $\eta^{L(R)}=1(0)$, if the bias is applied at the left lead, and vice versa.
Using Eq. (4) and matching wave functions at the scattering region boundary $(m=-1,1)$, we solve for $t_{\sigma\left(\sigma^{\prime}\right)}$ and then calculate transmission coefficient through the CNT. If the number of electrons in the dot is even, we obtain

$$
t_{\sigma}=\frac{-i 2 t_{0} \sin \left(k^{L}\right) S_{L R}^{\sigma}}{\left(t_{0} e^{-i k^{R}}-S_{R R}^{\sigma}\right)\left(t_{0} e^{-i k^{L}}-S_{L L}^{\sigma}\right)-S_{L R}^{\sigma} S_{R L}^{\sigma}}
$$

with

$$
S_{\lambda \lambda^{\prime}}^{\sigma}=\sum_{h>M} \frac{t_{h \sigma \sigma}^{\lambda^{*}} t_{h \sigma \sigma}^{\lambda^{\prime}}}{\epsilon-\xi_{h}-V_{g}-(4 M+1) \frac{e^{2}}{2 C}} ; \quad \lambda, \lambda^{\prime}=L, R .
$$

If the number of electrons is odd, we could not find an analytical solution in a closed form and we computed the transmission coefficient numerically. Nevertheless, we note that at low temperatures $\left(k_{B} T<\Delta E\right)$, the results reduce to transmission through a single state $(q=M+1)$ and the transmission matrix simplifies to the following form:

$$
\widetilde{\tilde{t}}=\frac{2 i}{\Delta} \sin \left(k^{L}\right) e^{i k^{L}} e^{i k^{R}} \times\left[\begin{array}{cc}
t_{q \uparrow}^{L^{*}} t_{q \uparrow}^{R} & t_{q \downarrow}^{L^{*}} t_{q \uparrow}^{R} \\
t_{q \uparrow}^{L^{*}} t_{q \downarrow}^{R} & t_{q \downarrow}^{L^{*}} t_{q \downarrow}^{R}
\end{array}\right],
$$

where $t_{q \uparrow(\downarrow)}^{L(R)}=t_{q \uparrow \uparrow(\downarrow \downarrow)}^{L(R)}+t_{q \uparrow \downarrow(\downarrow \uparrow)}^{L(R)}$ and

$$
\begin{aligned}
\Delta= & \sum_{\lambda=L, R}\left\{e^{i k^{L}} \sum_{\sigma, \sigma^{\prime}}\left|t_{\sigma, \sigma^{\prime}}^{\lambda}\right|^{2}\right\}-t_{0} \\
& \times\left[\epsilon-\xi_{q}-V_{g}-(4 M+3) \frac{e^{2}}{2 C}\right] .
\end{aligned}
$$

The conductance is calculated from the transmission coefficients,

$$
G=-\frac{e^{2}}{h} \sum_{N} \int d \epsilon \frac{\partial f}{\partial \epsilon}\left[T_{N}^{0}(\epsilon) F_{N}^{0}(\epsilon)+T_{N}^{1}(\epsilon) F_{N}^{1}(\epsilon)\right],
$$

where $F_{N}^{0(1)}$ describes the probability that the lowest $(N$ -1) states of the CNT are full and the $N$ th state has zero (one) electron; $T_{N}^{0}=\left|t_{\uparrow}\right|^{2}+\left|t_{\downarrow}\right|^{2}$ is evaluated from Eq. (5); and $T_{N}^{1}=\operatorname{tr}\left(\widetilde{\widetilde{t} \tilde{t}^{\dagger}}\right)$ is calculated from Eq. (6). The approach outlined here differs from previous theoretical works ${ }^{10,11}$ in that the different spin states are treated as being coupled rather than independent.

\section{TRANSMISSION COEFFICIENT}

Figure 1 illustrates, roughly, the physics contained in our model presented above where the spin degenerate state is represented using two close-by dashed lines. Below the lowest dashed line all the $M$ states are filled by electrons already inside the CNT region. For the case of even number of electrons already inside [Fig. 1(a)], an extra electron tunnels in and occupies one spin state pushing the other spin state to a charging energy $U$ higher; this electron then tunnels out to the right lead. For this case, there are two degenerate states which couple to the left lead, and only one state couples to 

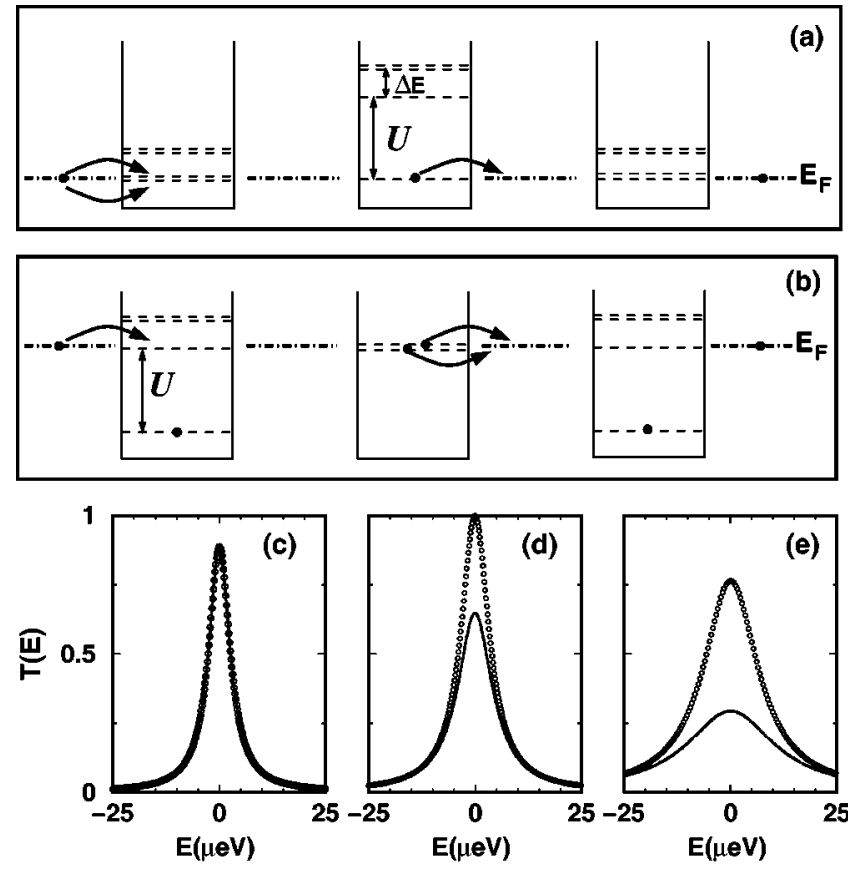

FIG. 1. Schematical plot of the tunneling and energy relaxation process for the even (a) and odd (b) number of electron cases. The double dashed lines indicate a spin degenerate state. $\Delta E$ is typical energy level spacing and $U$ is the charging energy. The black dots indicate an electron. (c), (d), and (e) are transmission coefficients $T(E)$ versus energy $E$, the solid line is for the odd case and the open circles are for the even case. (c) Symmetric coupling with $t^{L}=t^{R}$ $=0.001 \mathrm{eV} ; \quad$ (d) $t^{L}=0.001 \mathrm{eV}, \quad t^{R}=0.0014 \mathrm{eV} ; \quad$ (e) $t^{L}$ $=0.001 \mathrm{eV}, t^{R}=0.0024 \mathrm{eV}$.

the right lead. Therefore, for the even case, the transport "bottleneck' is the right tunnel barrier. Now consider the case of having an odd number of electrons already inside the CNT, as shown in Fig. 1(b) where one of the spin states is already occupied and an extra electron can only tunnel to the other state by overcoming the charging energy $U$. Once inside and after full relaxation of the energy landscape, the two electrons occupy the double spin-degenerate state and afterwards one of them tunnels out to the right lead. Hence for the odd case there is only one spin state which couples to the left lead and there are two spin states which couple to the right lead because either electron can tunnel out. Therefore, for the odd case, the transport bottleneck is the left tunnel barrier. We thus conclude that depending on the transport bottleneck, which in our theory is modeled by the coupling constants $t^{L(R)}$, there should be an odd-even asymmetry in the Coulomb peak heights. Moreover, if $t^{L}<t^{R}$ the peak heights for odd case will always be lower than those of the even case. For $t^{L} \sim t^{R}$ the odd peaks will still be lower due to contributions of the thermal probabilities $\left(F_{N}^{0}\right.$ and $\left.F_{N}^{1}\right)$ in Eq. (8). But for $t^{L}$ much larger than $t^{R}$ the odd peak can become higher than the even peak and in that case the peak heights alternation is reversed. The picture just presented is drastically simplified for the ease of discussing the basic idea. Our theory, however, includes other effects such as interference of the degenerate states and our numerics contains a contribution of multiple levels.
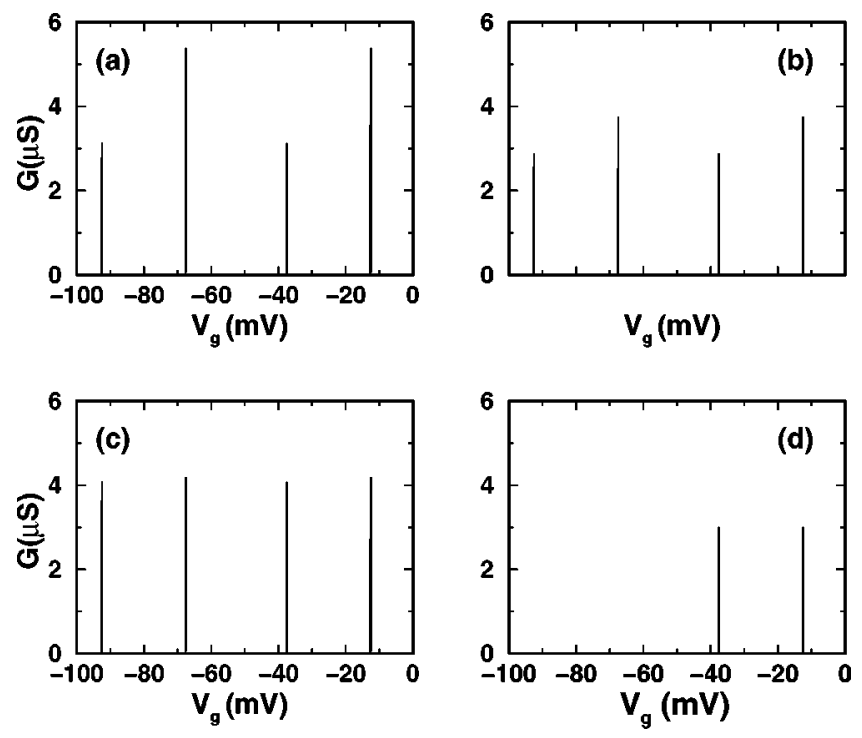

FIG. 2. Conductance $G$ as a function of $V_{g}$ for a CNT device. Parameters are: $\Delta E=5 \mathrm{meV}, U=25 \mathrm{meV}$, temperature $T$ $=100 \mathrm{mK}$. (a) $t^{L}=0.001 \mathrm{eV}, t^{R}=0.0014 \mathrm{eV}$; (b) $t^{L}=t^{R}$ $=0.001 \mathrm{eV}$; and (c) $t^{L}=0.0014 \mathrm{eV}, t^{R}=0.001 \mathrm{eV}$; Calculations were done with our new approach [Eq. (8)]. (d) $t^{L}=t^{R}=4$ $\times 10^{-6} \mathrm{eV}$, where Eq. (12) is used and $t^{L, R} \ll K_{b} T$ as required by the approach of Ref. 11.

As a check to our numerical calculations, we have verified that the following two situations give precisely the same transmission coefficients: (a) for the even case the incoming electron is from the left lead; (b) for the odd case the incoming electron is from the right lead. Since in both situations there are two states coupling to the left lead and one coupling to the right lead, they must give the same transmission coefficients. This is a very strong confirmation to our theory and numerics because for (a) and (b) the calculations use a completely different formula, i.e., using Eq. (5) for the even case, and using numerical solutions directly for the odd case, as discussed in the last section.

Figure 1(c) shows the transmission coefficients for a QD with even (odd) number of electrons presented by circles (solid line) as a function of energy when the coupling is symmetric. Even though the transmission coefficients are identical (as expected), conductance calculated using Eq. (8) shows an odd/even effect due to the statistical distribution function. When $t^{L}<t^{R}$, we show the corresponding results in Figs. 1(d) and (e); the transmission coefficients for an even number of electrons case is larger and this enhances the peak height alternation in the conductance as described later.

\section{ZERO-BIAS CONDUCTANCE}

To compare our results with the experimental data, we calculated zero-bias conductances of the CNT as a function of gate voltage $V_{g}$ at temperature $T=100 \mathrm{mK}$ using our model [Eq. (8)]. Figure 2(a) shows our numerical result for $t^{L}=0.001 \mathrm{eV}$ and $t^{R}=0.0014 \mathrm{eV}$, where a clear asymmetry of Coulomb peak heights is seen with the lower peaks corresponding to devices with an odd number of electrons already inside, and the higher peaks are for the even number 
cases. This result is qualitatively very similar to that of the experimental measurements. ${ }^{3}$ Figure 2(b) shows the situation for $t^{L}=t^{R}=0.001 \mathrm{eV}$ where the peak heights alternation persists but is somewhat reduced. When $t^{R}=0.001 \mathrm{eV}$ and $t^{L}=0.0014 \mathrm{eV}$, as shown in Fig. 2(c) the odd-even peak heights alternation becomes much less pronounced. However, if the bias voltage is reversed the differences in the peak heights reappears for the case of Fig. 2(c), and results identical to that in Fig. 2(a) are recovered.

In comparing these results to the experimental data, ${ }^{3}$ it must be emphasized that our model contains no randomness in the coupling constants of CNT to the leads, which is why we obtain a peak pattern with a perfect periodicity of two accounting for the even/odd number of electrons in the CNT. Such randomness is, however, possibly a general feature of experiments and can account for some of the further variations in the peak heights. For instance, the odd (even) experimental peaks, although all low (high), do not have exactly the same heights. This feature is possibly due to randomness in the coupling constants. Also, if the coupling of the CNT to the leads is large, ${ }^{13}$ it would be impossible to resolve the different peak height characteristics because, $\langle\Delta n\rangle$, the fluctuations in the number of electrons in the CNT would be large, and both types of processes would take place simultaneously. Other experimental difficulties that may prohibit these observations could include spin degeneracy liftoff and thermal excitations. While the latter should be controllable by decreasing the temperature, the former may be difficult to handle especially when combined with random coupling effects. Since the gate voltage alters the characteristics of the whole system, these two effects can lead to nonuniform coupling of the CNT to the leads. The peak heights would then be slightly randomized, making it difficult to distinguish between an odd and even number of electrons in the CNT region.

We have also attempted to understand the odd/even behavior within the familiar rate equation approach to Coulomb blockade. ${ }^{11}$ The simplest extension would be to modify the rate equation to include spin degeneracy. For simplicity of discussion, let us consider the case of a QD with a single state. At low $T, K_{b} T \ll \Delta E$, transmission occurs through independent eigenstates and therefore considering a single state is a valid approximation. We can represent the electron occupancy of this state as $n_{00}, n_{01}, n_{10}$, and $n_{11}$, where 0 and 1 correspond to a spin state being empty or filled. In the case of an empty quantum dot, $n_{11} \rightarrow 0$, and the rate equation can be easily solved leading to

$$
\begin{gathered}
n_{00}=t_{\text {out }}^{0} /\left(t_{\text {out }}^{0}+2 \times t_{\text {in }}^{0}\right) \\
n_{01}=n_{10}=t_{\text {in }}^{0} /\left(t_{\text {out }}^{0}+2 \times t_{\text {in }}^{0}\right) .
\end{gathered}
$$

However, considering the case of a QD with one electron (e.g., odd number of electrons), we get $n_{00} \rightarrow 0$ and the rate equation results in

$$
\begin{gathered}
n_{11}=t_{\text {in }}^{1} /\left(t_{\text {in }}^{1}+2 \times t_{\text {out }}^{1}\right) \\
n_{01}=n_{10}=t_{\text {out }}^{1} /\left(t_{\text {in }}^{1}+2 \times t_{\text {out }}^{1}\right),
\end{gathered}
$$

where

$$
\begin{aligned}
t_{\text {in }}^{k}=\Gamma^{L} \times & f^{L}\left(k, V_{b}, V_{g}\right)+\Gamma^{R} \times f^{R}\left(k, V_{b}, V_{g}\right) \\
t_{\text {out }}^{k}= & \Gamma^{L} \times\left[1-f^{L}\left(k, V_{b}, V_{g}\right)\right] \\
& +\Gamma^{R} \times\left[1-f^{R}\left(k, V_{b}, V_{g}\right)\right]
\end{aligned}
$$

are the rates for the electrons to enter and leave a QD with $k$ electrons at bias and gate voltages $V_{b}$ and $V_{g}$ with respect to the Fermi energy of the leads; and $f^{L, R}$ are the Fermi functions. $\Gamma^{L,(R)}$ is the tunneling rate between the left (right) reservoir and the QD eigenstate such that $h \Gamma^{L,(R)}=t^{L,(R)}$. In general $t_{\text {in }}^{k} \neq t_{\text {out }}^{k}$, therefore due to the difference in the denominators of Eqs. (9) and (10), the occupation numbers are different depending on whether there is an odd or an even number of electrons inside the QD. This seems to suggest a difference in the $\mathrm{CB}$ peak heights between odd and even cases even for situations involving symmetric coupling. To confirm if this is indeed the case, we have derived the zerobias conductance which is given by the following expression:

$$
\begin{aligned}
G= & 2 \frac{e^{2}}{h}\left(K_{b} T\right)^{-1} t^{L} t^{R} /\left(t^{L}+t^{R}\right) \\
& \times\left\{\frac{e^{a}}{\left(1+e^{a}\right)\left(2+e^{a}\right)}+\frac{e^{b}}{\left(1+e^{b}\right)\left(1+2 e^{b}\right)}\right\},
\end{aligned}
$$

where $a=\left(K_{b} T\right)^{-1}\left(\xi_{0}+e V_{g}+e^{2} / 2 C-E_{F}\right)$ and $b$ $=\left(K_{b} T\right)^{-1}\left(\xi_{0}+e V_{g}+3 e^{2} / 2 C-E_{F}\right)$, with $\xi_{0}$ being the energy eigenstate in the QD. Using this result, we plot in Fig. 2(d) the zero-bias conductance of a symmetrically coupled QD as a function of $V_{g}$. From this result it is clear that although the simple generalization of the rate equation results in different occupation numbers for an even or odd number of electrons for general situations, such a generalization is not enough to resolve odd-even behavior of conductance peak heights at the Coulomb blockade resonances. The reason is that at exactly the $\mathrm{CB}$ resonance, the Fermi functions in Eq. (11) are equal to $1 / 2$, making $t_{\text {in }}^{k}=t_{\text {out }}^{k}$ at the CB peak positions. In fact, even for an asymmetric coupling $\left(t^{L} \neq t^{R}\right)$, the simple generalization of the rate equation will predict identical peak heights for even and odd cases for any QD configuration. Therefore the simple generalization of the rate equation approach just presented does not give the even/ odd feature in the conductance. This exercise suggests that to resolve the experimental feature of the even/odd phenomenon, the rate equation approach, perhaps, should explicitly include spin degeneracy, i.e., $n_{01}$ and $n_{10}$ are indistinguishable.

\section{ASYMMETRIC I-V CHARACTERISTICS}

We have also investigated the $I-V_{b}$ characteristics of the CNT device at two different gate voltages as shown in Fig. 3, corresponding to an odd (solid line at $V_{g}=-37.478 \mathrm{mV}$ ) and even (dotted line at $V_{g}=-67.478 \mathrm{mV}$ ) number of electrons in the CNT region. These curves were obtained assuming that the voltage drops completely at the CNT-lead inter- 


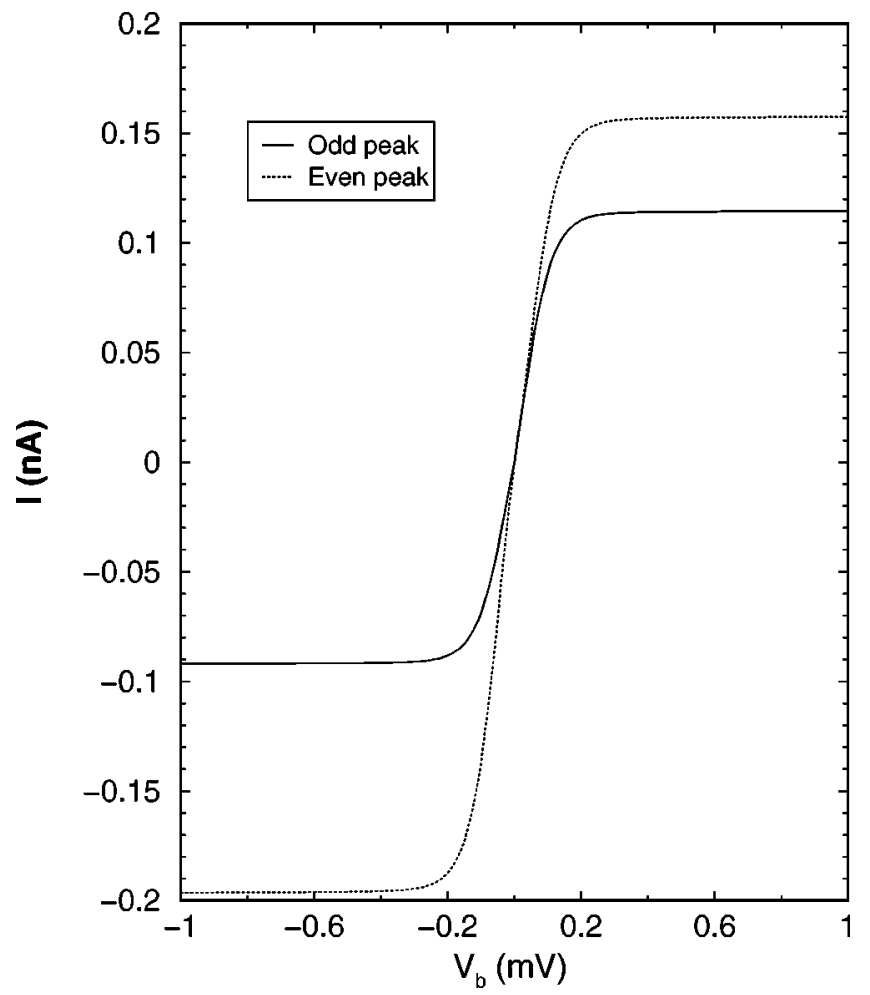

FIG. 3. $I$ vs $V_{b}$ for $V_{g}=37.478 \mathrm{mV}$ (solid line) and $V_{g}$ $=67.478 \mathrm{mV}$ (dotted line) at temperature $0.5 \mathrm{~K}$. CNT device parameters are $\Delta E=5 \mathrm{meV}, U=25 \mathrm{meV}, t^{L}=1 \mathrm{meV}$, and $t^{R}$ $=1 \mathrm{meV}$.

face, which is a reasonable assumption given the small bias voltages considered $(<1 \mathrm{mV})$ and the weak coupling between the CNT and the leads. As a function of bias voltage, the current increases linearly and then saturates. The rate of saturation is temperature dependent, and results are shown for $T=0.5 \mathrm{~K}$. This saturation effect which indicates the typical CB behavior is well known, and corresponds to a maximum tunneling per state. If a higher bias is applied [i.e., $>(U+\Delta E)]$, a new step would be expected to emerge. Note that there is a pronounced asymmetry in the saturation curves, which is due to the two effects already discussed above: the relaxation of the eigenstates and the asymmetry of the couplings. To interpret our data, we note that the bottle- neck for the current is the probability of entering (leaving) the CNT region if the number of electrons is odd (even). At $V_{g}=37.478 \mathrm{mV}$ (solid line in Fig. 3), the probability of entering is $\propto\left|t^{L}\right|^{2}$ for $I_{-}$and $\left|t^{R}\right|^{2}$ for $I_{+}$, resulting in $I_{+} / I_{-}$ $>1$ for $t^{R}>t^{L}$. Here $I_{-}, I_{+}$are saturation currents for negative and positive bias voltages, respectively. At $V_{g}$ $=67.478 \mathrm{mV}$ (dotted line in Fig. 3) there is an even number of electrons in CNT, we have the probability for the tunneling electron to leave CNT to be $\propto\left|t^{R}\right|^{2}$ for $I_{-}$and $\left|t^{L}\right|^{2}$ for $I_{+}$, leading to $I_{+} / I_{-}<1$. These numerical results are consistent with the experimental observations. ${ }^{3,14}$

So far our calculations assumed full eigenstate relaxation (so that Fig. 1 is true), and this is appropriate for weak coupling between the CNT and the leads so that electron dwell time inside the CNT region is large compared with the electron-electron relaxation time. If we do not assume full relaxation, our theory then predicts even peak heights a factor of 2 or more compared with the odd peak heights for symmetric coupling, and for a symmetric $I-V_{b}$ curve as the bias voltage is reversed (which is in disagreement with experimental observation). The enhancement of peak heights is, to a large extent, due to two spin states coupling to both the left and right leads for the even number of electrons case when relaxation is ignored.

\section{SUMMARY}

In summary, we have developed a formalism for the calculation of transmission coefficient through a quantum dot in the $\mathrm{CB}$ regime which treats the spin degenerate eigenstates separately. By assuming full eigenstate relaxation our theory provides a possible explanation to the new experimental features of high versus low conductance peaks for an even/odd number of electrons in CNT devices in the $\mathrm{CB}$ regime.

\section{ACKNOWLEDGMENTS}

We gratefully acknowledge correspondence with Professor Shi-Jie Xiong concerning their work; and financial support from NSERC of Canada and FCAR of Quebec (H.G.); RGC grant (HKU 7215/99P) from the Hong Kong SAR (J.W.); ONR N00014-98-1-0597 and NASA NAG8-1479 (C.R.).
${ }^{1}$ S.J. Tans, M.H. Devoret, H. Dai, A. Thess, R.E. Smalley, L.J. Geerligs, and C. Dekker, Nature (London) 386, 474 (1997).

${ }^{2}$ M. Bockrath, D.H. Cobden, P.L. McEuen, N.G. Chopra, A. Zettl, A. Thess, and R.E. Smalley, Science 275, 1922 (1997).

${ }^{3}$ David H. Cobden, M. Bochkrath, P.L. McEuen, A.G. Rinzler, and R.E. Smalley, Phys. Rev. Lett. 81, 681 (1998).

${ }^{4}$ L. Chico, L.X. Benedict, S.G. Louie, and M.L. Cohen, Phys. Rev. B 54, 2600 (1996); M. Buongiorno Nardelli, ibid. 60, 7828 (1999); A. Rochefort, Ph. Avouris, F. Lesage, and D. Salahub, ibid. 60, 13824 (1999).

${ }^{5}$ H. Mehrez, J. Taylor, H. Guo, J. Wang, and C. Roland, Phys. Rev. Lett. 84, 2682 (2000); C. Roland, M. Buongiorno Nardelli,
J. Wang, and H. Guo, ibid. 84, 2921 (2000); D. Orlikowski, H. Mehrez, J. Taylor, H. Guo, J. Wang, and C. Roland, Phys. Rev. B 63, 155412 (2001).

${ }^{6}$ U. Meirav, M.A. Kastner, and S.J. Wind, Phys. Rev. Lett. 65, 771 (1990).

${ }^{7}$ D. Goldhaber-Gordon, Hadas Shtrikman, D. Mahalu, David Abusch-Magder, U. Meirav, and M. A. Kastner, Nature (London) 391, 156 (1998).

${ }^{8}$ In Fig. 2(d) of Ref. 6, all the six Coulomb peaks can be arranged as a low-high alternation sequence. Similarly we observe a highlow-high sequence in Fig. 2(c) of Ref. 7. We note that, in all these experiments, the coupling of the quantum dots to leads 
were very weak as indicated by very small conductances in the data. Hence it is likely that in these meaurements the electron number fluctuation inside the dots was small.

${ }^{9}$ For a review, see (Z. Phys. B 85, 317 (1991); Single Charge Tunneling, edited by H. Grabert and M.H. Devoret (Plenum Press, New York, 1992); Marc A. Kastner, Phys. Today 46(1), 24 (1993); R.C. Ashoori, Nature (London) 379, 413 (1996).

${ }^{10}$ Yigal Meir, Ned S. Wingreen, and Patrick A. Lee, Phys. Rev. Lett. 66, 3048 (1991).
${ }^{11}$ C.W.J. Beenakker, Phys. Rev. B 44, 1646 (1991).

${ }^{12}$ Shie-Jie Xiong and Ye Xion, Phys. Rev. Lett. 83, 1407 (1999).

${ }^{13}$ Sera M. Cronenwett, Tjerk H. Oosterkamp, and Leo P. Kouwenhoven, Science 281, 540 (1998).

${ }^{14} \mathrm{~A}$ discrepancy between our calculations and the experimental results (Ref. 3) is that our calculations give a clear saturation plateau, while the experimental curves show a linear increase with a small slope. This difference is most likely due to a lowering of the tunnel barriers at the lead/CNT interface with increasing bias voltage. This effect is absent in our model. 